\title{
А.Ф. Киосов
}

Областная клиническая больница № 2, Челябинск, Российская Федерация

\section{Вопросы оценки постнатального роста недоношенных детей}

\section{Часть 1. Графики роста недоношенных детей}

\author{
Contacts: \\ Kiosov Andrey Fyodorovich, Candidate of Medical Science, Head of the Department for premature infants (2nd stage) at the Regional Clinical \\ Hospital SBIH No. 2 \\ Address: 18 Gagrin Str., Chelyabinsk 454010, Tel.: (351) 256-01-28, e-mail: kiosow@mail.ru \\ Article received: 12.06 .2013 , Accepted for publication: 23.12 .2013
}

Оценка роста недоношенных детей имеет существенное значение в неонатологии и педиатрии. Она производится с учетом корригированного возраста недоношенного. Коррекцию осуществляют в первые 2 года жизни. Это особенно важно для детей, рожденных до 32-33-й нед гестации и весом менее 1500 г. Оценку выполняют с использованием диаграмм роста для недоношенных детей. Кривые роста содержат 3 измерения: вес, рост и окружность головы. В настоящее время существует несколько кривых роста. Более 40 лет используют кривые Любченко (1963), кривые Бэбсона и Бенда (1976), однако они неточно отражают рост недоношенного. В последние годы на основании больших групп детей с учетом половых различий разработаны современные кривые роста. Фентоном (2003) предложены диаграммы роста для недоношенных детей. Они представляют собой графики процентилей веса, роста, окружности головы. График позволяет сравнить рост недоношенного с ростом плода, начиная с 22-й нед гестации и до 10-й нед постнатального возраста. Гендерные различия оказывают существенное влияние на показатели роста детей: мальчики имеют больший вес, рост и окружность головы. Фентоном (2013) созданы кривые роста для девочек и мальчиков. При оценке роста плода и ребенка после рождения также необходимо учитывать национальные, расовые различия. Кривые роста плода и недоношенного ребенка имеют большое значение для оценки популяционного здоровья и оценки индивидуального роста. Их применяют для оценки показателей ребенка при рождении и для оценки динамики роста после рождения. Кривые роста используют в отделениях интенсивной терапии и в педиатрических отделениях. Для достижения адекватного роста необходимо проводить коррекцию режима, ухода, питания ребенка.

Ключевые слова: недоношенные дети, корригированный возраст, графики роста, темп роста, гендерные различия.

(Вопросы современной педиатрии. 2013; 12 (6): 82-84)

Вопрос оценки параметров роста и физического развития у недоношенных детей является важным в неонатологии и педиатрии. По-прежнему продолжаются споры по поводу того, как быстро должны расти недоношенные дети. Всегда существует баланс между слишком быстрой и слишком медленной прибавкой в весе. Низкая при- бавка в весе и быстрый набор массы тела могут предрасполагать к развитию болезней [1]. Физическое развитие детей имеет огромное социальное и медицинское значение. Эксперты Всемирной организации здравоохранения определяют показатели физического развития как один из основополагающих критериев в комплекс-

\section{A.F. Kiosov}

Regional Clinical Hospital № 2, Chelyabinsk, Russian Federation

\section{Questions of Evaluation of Postnatal Growth in Preterm Children}

\section{Part 1. Growth Charts of Premature Babies}

Evaluation of the growth of premature children is of great importance in neonatology and pediatrics. Evaluation of the growth is carried out taking into account the corrected age of preterm infants. Correction is performed during the first two years of life. This is especially important for children born before 32-33 weeks of gestation and weighing less than 1500 g. Evaluation of the growth is carried out with the use of growth charts for premature babies. Growth curves contain three measurements: weight, height and head circumference. Today, there are several growth curves. Lubchenco curves (1963), the curves of Babson and Benda (1976) have been used for more than forty decades. Currently, they do not accurately reflect the growth of a premature baby. In recent years, modern growth curves have been developed. Modern curves are developed on the basis of larger groups, taking into account gender differences. Fenton (2003) proposed growth charts for premature babies. They consist of graphs of percentiles of weight, height and head circumference. Graph compares the growth of preterm infants with fetal growth from 22 weeks to 10 weeks of postnatal age. Gender differences have a significant impact on the growth of children. Fenton (2013) established growth curves for girls and boys. Boys have higher weight, height and head circumference than girls. In assessing the growth of the fetus and the baby after birth national and racial differences should be taken into account. The growth curves of the fetus and preterm infant are important for population health assessment and evaluation of individual growth. The growth curves are used to evaluate the child's performance at birth and for the assessment of the growth of the baby after birth. The growth curves are used in intensive care and pediatric units. In order to achieve adequate growth it is necessary to carry out the correction of regimen, nursing and nutrition of a child. Key words: premature babies, corrected age, Growth charts, growth rate, gender differences.

(Voprosy sovremennoi pediatrii - Current Pediatrics. 2013; 12 (6): 82-84) 
ной оценке состояния здоровья ребенка [2]. Ребенок, который прибавляет в весе так, как должен, практически всегда здоров [1].

\section{ГЕСТАЦИОННЫЙ, ПОСТНАТАЛЬНЫЙ, ПОСТКОНЦЕПЦИОННЫЙ И КОРРИГИРОВАННЫЙ ВОЗРАСТ НЕДОНОШЕННОГО РЕБЕНКА}

При оценке физического развития у недоношенных детей следует учитывать такие понятия, как гестационный, постнатальный, постконцепционный и корригированный возраст. Под гестационным возрастом принято понимать число полных недель, прошедших между первыми сутками последней менструации и датой родов. Постнатальный возраст - это фактический (календарный) возраст, т.е. число месяцев, прошедших после рождения ребенка. Постконцепционный (постменструальный) возраст рассчитывается как сумма срока гестации и постнатального возраста ребенка. Для расчета корригированного возраста необходимо из календарного возраста отнять те недели, на которые раньше срока родился недоношенный $[3,4]$. Физическое развитие недоношенных детей необходимо оценивать только по корригированному возрасту. Это особенно важно для детей, рожденных до 32-33-й нед беременности и весом менее 1500 г. Для детей, рожденных в 32-33 нед или позже, коррекция гестационного возраста может завершаться в возрасте 1 года. Корригированный возраст у недоношенных детей должен рассчитываться в первые два года жизни. Некоторые авторы предлагают проводить коррекцию до 3 или 7 лет. Момент завершения корректировки возраста должен быть обязательно зафиксирован [3-5].

\section{ГРАФИКИ РОСТА ПЛОДА}

\section{И НЕДОНОШЕННОГО РЕБЕНКА}

Для оценки роста в неонатологии используют кривые роста плода и недоношенного ребенка. Кривые роста представляют собой графическое отображение динамики показателей антропометрии в зависимости от срока гестации [6]. Кривые роста, как правило, содержат 3 измерения: вес, рост и окружность головы. Измерение веса, по мнению экспертов Всемирной организации здравоохранения, является точным измерением, поскольку для этого используют электронные весы [7]. Окружность головы также может быть измерена с высокой степенью точности. Рост ребенка, по мнению экспертов, может быть измерен менее точно из-за вопросов позиционирования [7-10].

В настоящее время существуют различные кривые оценки роста плода и недоношенного ребенка. Уже несколько десятилетий специалисты применяют кривые Любченко (1963), кривые Бэбсона и Бенда (1976). Кривые Любченко содержат процентили кривых веса, длины, окружности головы и весового индекса (индекс пропорциональности тела) [11]. Кривые Бэбсона и Бенда отображают стандартные отклонения веса, роста, окружности головы [12]. По данным опроса неонатологов, опубликованного в 2003 г., 50\% медработников до сих пор пользуются кривыми Бэбсона и Бенда, 42\% специалистов - кривыми Любченко [13]. Нормограммы Любченко, Бэбсона и Бэнда были созданы более 40 лет назад, и сейчас не могут адекватно отражать рост недоношенного ребенка. Кроме того, эти кривые имеют существенные ограничения из-за маленьких размеров выборки, неоднородности данных, отсутствия учета пола. В 1960-х гг. глубоконедоношенных детей рождалось значительно меньше. Недоношенные дети имели более высокие показатели смертности, и поэтому глубоконедо- ношенные дети не были включены в указанные кривые роста. Текущее использование этих кривых приводит к переоценке числа младенцев, больших по размеру для конкретного срока гестации, и недооценивают число маленьких детей [14].

Взамен прежних и с учетом последних данных были разработаны новые кривые роста. К современным кривым относятся кривые Фентона. При наложении на старые новые кривые роста обычно демонстрируют схожие параметры. Однако имеются и достоверные различия. Современные кривые роста отличаются от старых тем, что дети до 30 нед гестации имеют более низкие показатели, чем сообщалось ранее; у новорожденных старше 36 нед гестации показатели более высокие. Указанные кривые более точно отражают темпы роста и являются важными критериями для оценки размера ребенка после рождения и оценки послеродового роста ребенка. Прежние кривые не могут отражать темпы роста детей в настоящее время. Современные центильные кривые разработаны на основании больших групп выборки, с учетом гендерных различий [14]. Чем больше размер выборки, тем больше уверенность в крайнем процентиле. Однако, даже используя эти кривые роста, 3-й и 97-й перцентиль на них на сроке 23 нед следует интерпретировать осторожно, т. к. в этом возрасте размер выборки не очень велик $[14,15]$.

Современные кривые роста Фентона (2003, 2013) могут быть использованы для мониторинга роста плода и недоношенного ребенка. Кривые Фентона включают в себя 3, 10, 50, 90 и 97-й перцентиль веса, роста, окружности головы, которые нанесены на сетку. В зоне от 10-го до 90-го перцентиля располагаются средние показатели физического развития, свойственные $80 \%$ недоношенных детей [16]. В зонах от 10-го до 3-го и от 90-го до 97-го перцентиля находятся величины, свидетельствующие об уровне развития ниже или выше среднего, свойственные только 7\% условно здоровых недоношенных. Величины, находящиеся ниже 3-го и выше 97-го перцентиля, - это области очень низких и очень высоких показателей, которые встречаются у здоровых недоношенных не чаще, чем в 3\% случаев. График роста Фентона имеет крупный масштаб, что обеспечивает высокую точность. Шаг веса ребенка составляет 100 г, шаг роста и окружности головы -1 см. Используется интервал времени 1 нед. График позволяет сравнить рост недоношенного ребенка с ростом плода, начиная с 22 нед гестации и до 10 нед постнатального возраста [13]. Диаграмма специально продлена до 50 нед, поскольку большинство недоношенных детей выписывают домой именно к этому возрасту [16]. В нижней части диаграммы сделано пространство для отметки данных измерений. Хотя Фентоном в 2003 г. были обнаружены различия между мальчиками и девочками, оба пола были усреднены и помещены в одну диаграмму роста [13]. В 2013 г. Фентоном были созданы новые нормограммы роста отдельно для плодов женского и мужского пола [17].

Кривые роста плода и недоношенного ребенка имеют важное значение для оценки общественного популяционного здоровья и индивидуального роста ребенка. Эпидемиологические исследования с использованием кривых роста направлены на сравнение роста детей в разных популяциях, группах [18]. Антропометрические показатели могут отличаться в зависимости от пола и национальности. Гендерные и расовые особенности оказывают существенное воздействие на показатели роста. Существование различий может иметь разные объяснения: генетические причины, социальноэкономический статус, уровень медицинской помощи. 
О различиях показателей роста в зависимости от пола было известно давно. Ранее гендерные различия не считались достаточно важными и не учитывались при составлении центильных графиков роста. В дальнейшем были найдены достоверные статистические различия между девочками и мальчиками на всех сроках гестации. Недоношенные мальчики в сравнении с девочками имеют достоверно высокие показатели веса, роста и окружности головы. Например, различия в массе тела на сроках гестации от 23 до 40 нед могут варьировать от 40 до 180 г. Наибольшие различия между разными полами регистрируют в конце беременности [19]. Это подтверждает необходимость создания отдельных графиков роста для мальчиков и девочек. Фентон в 2013 г. представил центильные кривые роста для мужского и женского пола [17]. При сравнении мужской и женской кривой роста выявлено, что младенцы мужского пола имеют больший вес при рождении на 2,8-9,1\% в зависимости от срока гестации. Мальчики также имеют длину тела больше на 1,2-2,0\% и окружность головы больше на 1,3-2,7\%, тоже в зависимости от срока гестации [14]. Гендерные различия наглядно демонстрируются графически на кривых роста. Гендерные кривые необходимы для предотвращения неправильной оценки младенцев мужского пола на соответствие сроку гестации (малый, соответствующий и большой для гестационного возраста). Без учета гендерных различий большое число мальчиков будет неправильно классифицировано как "соответствующий сроку гестации" вместо "малый для срока гестации". При оценке роста плода и ребенка после рож-

\section{REFERENCES}

1. Nedonoshennyi rebenok. Esli rebenok rodilsya ran'she sroka. Per. s norv. Pod red. prof. E. N. Baibarinoi [Premature. If an infant was born earlier. Translated from Norway. Edited by E. N. Baibarina, professor]. Moscow, GEOTAR-Media, 2012. 192 p.

2. World Health Organization. Physical status: the use and interpretation of anthropometry. Report of a WHO Expert Committee. World Health Organ Tech Rep Ser. 1995; 854: 1-452. 3. Elliman A. M., Bryan E. M., Elliman A.D., Harvey D. R. Gestational age correction for height in preterm children to seven years of age. Acta Paediatr. 1992; 81: 836-839.

4. Wang Z., Sauve R.S. Assessment of postneonatal growth in VLBW infants: selection of growth references and age adjustment for prematurity. Can. J. Public Health. 1998; 89: 109-114.

5. Guo S.S., Roche A.F., Chumlea W. C., Casey P.H., Moore W.M. Growth in weight, recumbent length, and head circumference for preterm low-birthweight infants during the first three years of life using gestation-adjusted ages. Early Hum. Dev. 1997; 47: 305-325.

6. Dancis J., O'Connell J. R., Holt L. E. A grid for recording the weight of premature infants. J. Pediatr. 1948; 33: 570-572.

7. WHO Expert Committee. The newborn infant. In Physical Status: The Use and Interpretation of Anthropometry. Geneva: World Health Organization. 1995. P. 161-262.

8. Engstrom J. L., Kavanaugh K., Meier P.P., Boles E., Hernandez J., Wheeler D., Chuffo R. Reliability of in-bed weighing procedures for critically ill infants. Neonat. Netw. 1995; 14: $27-33$.

9. Sutter K., Engstrom J.L., Johnson T.S., Kavanaugh K., Ifft D.L. Reliability of head circumference measurements in preterm infants. Pediatr. Nurs. 1997; 23: 485-490.

10. Rosenberg S. N., Verzo B., Engstrom J. L., Kavanaugh K., Meier P.P. Reliability of length measurements for preterm infants. Neonat. Netw. 1992; 11: 23-27.

11. Lubchenco L. O., Hansman C., Boyd E. Intrauterine growth in length and head circumference as estimated from live births at gestational ages from 26 to 42 weeks. Pediatrics. 1966; 37: 403-408. дения необходимо учитывать национальные, расовые различия [19]. Наиболее значимые различия установлены при сравнении показателей европейской и афроамериканской расы. Белые младенцы имеют больший вес при рождении, чем черные младенцы (на 3-5\% в зависимости от гестационного возраста) [19, 20].

Кривые роста плода и ребенка применяют в неонатологии для оценки антропометрических показателей новорожденного [21]. На основании этого все новорожденные (доношенные и недоношенные) могут быть разделены на 3 большие группы: соответствующий сроку гестации (от 10-го до 90-го перцентиля), малый для срока гестации (ниже 10-го перцентиля), большой для гестационного возраста (больше 90-го перцентиля) [14, 18]. Кривые роста плода и недоношенного ребенка используют для оценки динамики роста ребенка после рождения. Для недоношенных детей "золотым стандартом" роста является внутриутробный рост плода [22]. Однако достичь внутриутробного темпа роста недоношенному ребенку после рождения не всегда удается [18]. У недоношенных детей это связанно с большой убылью массы тела после рождения, длительным ее восстановлением, нарушением процессов адаптации, метаболическими нарушениями, болезнями неонатального периода. Кривые роста также широко применяют в отделениях интенсивной терапии и в педиатрических отделениях. Они позволяют своевременно выделять группы риска и искать возможности коррекции роста. Своевременная коррекция режимных моментов, ухода, питания позволяет достичь адекватных темпов роста [23].

12. Babson S. G., Benda G. I. Growth graphs for the clinical assessment of infants of varying gestational age. J. Pediatr. 1976; 89: 814-820. 13. Fenton T.R. A new growth chart for pretermbabies: Babson and Benda's chart updatedwith recent data and a new format. BMC Pediatr. 2003; 3: 13-22.

14. Olsen I. E., Groveman S. A., Lawson M. L., Clark R. H., Zemel B.S. New intrauterine growth curves based on United States data. Pediatrics. 2010; 125: 214-224.

15. Kramer M.S., Morin I., Yang H., Platt R. W., Usher R., McNamara H., Joseph K.S., Wen S.W. Why are babies getting bigger? Temporal trends in fetal growth and its determinants. J. Pediatr. 2002; 141: 538-542.

16. Fenton T.R., McMillan D.D., Sauve R.S. Nutrition and growth analysis of very low birth weight infants. Pediatrics. 1990; 86: 378-383.

17. Fenton T.R., Kim J.H. A systematic review and meta-analysis to revise the Fenton growth chart for preterm infants. BMC Pediatrics. 2013. URL: http://www.biomedcentral.com/1471-2431/13/59.

18. Wrigth K., Dawson J. P., Fallis D., Vogt E., Lorch V. New postnatal growth grids for very low weight infants. Pediatrics. 1993; 91: 922-926.

19. Thomas P., Peabody J., Turnier V., Clark R.H. A new look at intrauterine growth and the impact of race, altitude, and gender. Pediatrics. 2000; 106 (2): 21.

20. Denham M., Schell L. M., Gallo M., Stark A. Neonatal size of low socioeconomic status Black and White term births in Albany County, NYS. Ann. Hum. Biol. 2011; 28 (2): 172-183.

21. Battaglia F.C., Lubchenco L.O. A practical classification of newborn infants by weight and gestational age. J. Pediatr. 1967; 71 (2): 159-163.

22. American Academy of Pediatrics, Committee on Nutrition. Nutritional needs of lowbirthweight infants. Pediatrics. 1977; 60 (4): 519-530.

23. Adamkin D. H. Enteral nutrition and postdischarge nutrition. Nutrition management of the very low-birthweight infant: II. Optimizing. NeoReviews. 2006; 7: 608-614. 